Proyecciones Journal of Mathematics

Vol. 35, No 4, pp. 457-468, December 2016.

Universidad Católica del Norte

Antofagasta - Chile

\title{
Stability of generalized Jensen functional equation on a set of measure zero
}

\author{
Hajira Dimou \\ University of Ibn Tofail, Morocco \\ Youssef Aribou \\ University of Ibn Tofail, Morocco \\ Abdellatif Chahbi \\ University of Ibn Tofail, Morocco \\ and \\ Samir Kabbaj \\ University of Ibn Tofail, Morocco \\ Received : June 2016. Accepted : August 2016
}

\begin{abstract}
Let $E$ is a complex vector space and $F$ is real (or complex) $B$ nach space. In this paper, we prove the Hyers-Ulam stability for the generalized Jensen functional equation

$$
\sum_{k=0}^{m-1} f\left(x+b_{k} y\right)=m f(x), x, y \in E,
$$

where $f: E \rightarrow F$ and $b_{k}=\exp \left(\frac{2 i \pi k}{m}\right)$ for $0 \leq k \leq m-1$, on a set of Lebesgue measure 0 .
\end{abstract}

Subjclass [2010] : Primary 39B82; Secondary 39B52.

Keywords : $K$ - Jensen functional equation, Hyers-Ulam stability. 


\section{Introduction}

The question concerning the stability of functional equations has been posed by S. M. Ulam in 1940 [29], and its solution given by H.D. Hyers [13] in 1941. Hyers theorem was generalized by T. Aoki [1] for additive mappings and by Th. M. Rassias [22] for linear mappings by considering an unbounded Cauchy difference. The paper [22] of Th. M. Rassias has provided a lot of influence in the development of what we now call Hyers-Ulam-Rassias stability of functional equations. During the last decades, many stability problems for various functional equations have been studied by numerous mathematicians, we refer, for example, to [12],[14],[15],[17].

The stability problems of several functional equations on a restricted domain have been extensively investigated by a number of authors, for example [[4], [9], [8], [10] [11], [17], [20], [26], [23] ].

It is very natural to ask if the restricted domain $D=\left\{(x, y) \in \mathbf{E}^{2}\right.$ : $\|x\|+\|y\| \geq d\}$ can be replaced by a much smaller subset $\Omega \subset D$ (e.g. a subset of measure 0 in a measure space $E$ ).

In 2013, J. Chung in [8] answered to this question by considering the stability of the Cauchy functional equation

$$
f(x+y)=f(x)+f(y)
$$

in a set $\Omega \subset\left\{(x, y) \in \mathbf{R}^{2}:\|x\|+\|y\| \geq d\right\}$ where $m(\Omega)=0$ and $f: \mathbf{R} \rightarrow \mathbf{R}$.

In 2014, J. Chung and J. M. Rassias [9] proved the stability of the quadratic functional equation in a set of measure zero.

Throughout we assume that $E$ is $\mathbf{C}$ vector space and $F$ vector space over $K=\mathbf{R}$ or $\mathbf{C}$ and $2 \leq m \in \mathbf{N}$. Our aim is to prove the Hyers-Ulam stability for the generalized Jensen functional equation

$$
\sum_{k=0}^{m-1} f\left(x+b_{k} y\right)=m f(x), x, y \in E,
$$

where $b_{k}=\exp \left(\frac{2 i \pi k}{m}\right)$ for $0 \leq k \leq m-1$, on a set Lebesgue measure 0 . These results are applied to study of an asymptotic behavior of this functional equation. The stability and solution of this equation and its generalizations were studied by numerous researchers, see for example [5, 2, 19, 28].

We also recall the following theorem which will be used in the sequel. 
Theorem 1.1 ([6]). Suppose that $E$ is a complex vector space and $F$ is real (or complex) Banach space, $m \geq 2 \in \mathbf{N}, b_{k}=\exp \left(\frac{2 i k \pi}{m}\right)$ for $0 \leq k \leq$ $m-1$ and $\delta \geq 0$. If $f: E \rightarrow F$ satisfies

$$
\left\|\sum_{k=0}^{m-1} f\left(x+b_{k} y\right)-m f(x)\right\| \leq \delta
$$

for all $x, y \in E$. Then there exists a unique generalized polynomial (GP) $q: E \rightarrow F$ at most $m-1$ such that $q(0)=0$ and

$$
\|f(x)-f(0)-q(x)\| \leq 3^{m-1} \delta \text { for all } x \in E .
$$

Moreover

$$
\sum_{k=0}^{m-1} q\left(x+b_{k} y\right)-m q(x)=0 \text { for all } x, y \in E \text {. }
$$

\section{Main result}

For given $x, y, t \in E$

$$
P_{x, y, t}=\left\{(x ; t),\left(x+b_{k} t ; y\right),\left(x+b_{k} y ; t\right), k=0, \ldots, m-1\right\} .
$$

Let $\Omega \subset E^{2}$. Throughout this section, we assume that $\Omega$ satisfies the condition:

For given $x, y \in E$ there exists $t \in E$ such that

$$
\text { (C) }\left\{(x, t),\left(x+b_{k} t, y\right),\left(x+b_{k} y, t\right)\right\} \subset \Omega .
$$

We prove the following stability theorem in $\Omega$.

Theorem 2.1. Let $\delta \geq 0$. Suppose $f: E \rightarrow F$ satisfies the functional inequality

$$
\left\|\sum_{k=0}^{m-1} f\left(x+b_{k} y\right)-m f(x)\right\| \leq \delta
$$

for all $(x, y) \in \Omega$, where $b_{k}=\exp \left(\frac{2 i k \pi}{m}\right), 0 \leq k \leq m-1$. Then there exists a unique generalized polynomial (GP) $q: E \rightarrow F$ of degree at most $m-1$ such that $q(0)=0$ and

$$
\|f(x)-f(0)-q(x)\| \leq 3^{m} \delta \text { for all } x \in E .
$$

Moreover

$$
\sum_{k=0}^{m-1} q\left(x+b_{k} y\right)-m q(x)=0 \text { for all } x, y \in E \text {. }
$$


Proof. Let $D(x, y)=\sum_{k=0}^{m-1} f\left(x+b_{k} y\right)-m f(x)$. It is clear that

$$
\begin{aligned}
m\left[\sum_{k=0}^{m-1}\left(f\left(x+b_{k} y\right)-m f(x)\right]\right. & =\left[\sum_{k=0}^{m-1}\left(m f\left(x+b_{k} y\right)-\sum_{n=0}^{m-1} f\left(x+b_{k} y+b_{n} t\right)\right)\right] \\
& +\left[\sum_{k=0}^{m-1}\left(-m f\left(x+b_{k} t\right)+\sum_{n=0}^{m-1} f\left(x+b_{k} t+b_{n} y\right)\right)\right] \\
& +m\left[\sum_{k=0}^{m-1} f\left(x+b_{k} t\right)-m f(x)\right] \\
& =-\sum_{k=0}^{m-1} D\left(x+b_{k} y, t\right) \\
& +\sum_{k=0}^{m-1} D\left(x+b_{k} t, y\right)+m D(x, t)
\end{aligned}
$$

Since $\Omega$ satisfies (C), for given $x, y \in E$, then there exists $t \in E$ such that $\left\|D\left(x+b_{k} y, t\right)\right\| \leq \delta,\left\|D\left(x+b_{k} t, y\right)\right\| \leq \delta$ and $\|D(x, t)\| \leq \delta$. Now by using the triangle inequality, we get

$$
m \| \sum_{k=0}^{m-1}\left(f\left(x+b_{k} y\right)-m f(x) \| \leq 3 m \delta, \quad x, y \in E .\right.
$$

This implies that

$$
\| \sum_{k=0}^{m-1}\left(f\left(x+b_{k} y\right)-m f(x) \| \leq 3 \delta, \quad x, y \in E .\right.
$$

Next, according to Theorem 1.1, there exists a unique generalized polynomial (GP) $q: E \rightarrow F$ of degree at most $m-1$ such that $q(0)=0$ and

$$
\|f(x)-f(0)-q(x)\| \leq 3^{m} \delta \text { for all } x \in E .
$$

This completes the proof.

Now with $m=2$. The condition $(C)$ is reduced to the following: for given $x, y \in E$ there exists $t \in E$ such that

$$
\{(x+y, t),(x-y, t),(x+t, y),(x-t, y),(x, t)\} \subset \Omega .
$$

As a direct consequence of Theorem 2.1, we obtain the following corollary 
Corollary 2.2. Let $\delta \geq 0$. Suppose that $f: E \rightarrow F$ satisfies the functional inequality

$$
\|f(x+y)+f(x-y)-2 f(x)\| \leq \delta \text { for all } x, y \in \Omega .
$$

then there exists a unique additive mapping $A: E \rightarrow F$ such that

$$
\|f(x)-f(0)-A(x)\| \leq 9 \delta \text { for all } x \in E .
$$

\section{The Ulam-Hyers stability in a set of Lebesgue measure zero}

In this section, we prove the Ulam-Hyers stability in a set of Lebesgue measure zero for the functional inequality

$$
\left\|\sum_{k=0}^{m-1} f\left(z+b_{k} \xi\right)-m f(z)\right\| \leq \delta \text { for all }(z, \xi) \in \Omega
$$

where $f: \mathbf{C} \rightarrow F$ and $\Omega \subset \mathbf{C}^{2}$ is of four-dimensional Lebesgue measure zero, and the Jensen functional inequality

$$
\|f(x+y)+f(x-y)-2 f(x)\| \leq \delta \text { for all }(x, y) \in \Omega \subset \mathbf{R}^{2},
$$

where $f: \mathbf{R} \rightarrow F$ and $\Omega$ is of two-dimensional Lebesgue measure zero.

Firstly, the inequality (3.1) is a particular case of(1.3) where $E=\mathbf{C}$. Now, the condition $(C)$ reduces to the following:

For given $z, \xi \in \mathbf{C}$ there exists $\eta \in \mathbf{C}$ such that

$$
\left\{\left(z+b_{k} \xi, \eta\right),\left(z+b_{k} \eta, \xi\right),(z, \eta): k=0, \ldots, m-1\right\} \subset \Omega .
$$

By virtue of Theorem 2.1, it suffice to construct a set $\Omega \subset \mathbf{C}^{2}$ of measure zero satisfying (3.3). It is known from [[21], Theorem 1.6] that there exists a set $K \subset \mathbf{R}$ of Lebesgue measure 0 such that $\mathbf{R} \backslash K$ is of first Baire category. That is, $\mathbf{R} \backslash K$ is a countable union of nowhere dense subsets of $\mathbf{R}$.

Lemma 3.1 (Lemma 2.4, [9]). Let $K$ be a subset of $\mathbf{R}$ of measure 0 such that $K=\mathbf{R} \backslash K$ is of first Baire category. Then, for any countable subsets $U \subset \mathbf{R}, V \subset \mathbf{R} \backslash\{0\}$ and $M>0$, there exists $t \geq M$ such that

$$
U+t V=\{u+t v: u \in U, v \in V\} \subset K .
$$


From now on we identify $\mathbf{C}$ with $\mathbf{R}^{2}$.

Theorem 3.2. Let $K$ be the set defined in Lemma 3.1, $R$ be the rotation

$$
\left(\begin{array}{cccc}
-\cos \theta & 0 & \sin \theta & 0 \\
0 & -\cos \theta & 0 & \sin \theta \\
-\sin \theta & 0 & -\cos \theta & 0 \\
0 & -\sin \theta & 0 & -\cos \theta
\end{array}\right)
$$

with $\theta \in \mathbf{R} \backslash\left\{\frac{\pi}{2}+k \pi, k \pi / k \in \mathbf{Z}\right\}$ and $\Omega=R^{-1}(K \times K \times K \times K)$. Then $\Omega$ satisfies (3.3) and has four-dimensional Lebesgue measure 0.

Proof. Our method in this proof is inspired by [10, Theorem 3.2]. Let

$$
z=x+i y, \quad \xi=u+i v, \quad \eta=t+i s \in \mathbf{C}, k=0,1, \cdots, m-1,
$$

and let

$$
\begin{aligned}
P_{z, \xi, \eta, k}=\{ & \left.\left(x+u \cos \frac{2 \pi k}{m}-v \sin \frac{2 \pi k}{m}, y+u \sin \frac{2 \pi k}{m}+v \cos \frac{2 \pi k}{m}, t, s\right)\right\} \\
& \bigcup\left\{\left(x+t \cos \frac{2 \pi k}{m}-s \sin \frac{2 \pi k}{m}, y+t \sin \frac{2 \pi k}{m}+s \cos \frac{2 \pi k}{m}, u, v\right),\right. \\
& (x, y, t, s)\} .
\end{aligned}
$$

Then $\Omega$ satisfies (3.3) if and only if, for every $z=x+i y$ and $\xi=u+i v \in$ $\mathbf{C}$, there exists $\eta=t+i s \in \mathbf{C}$ such that

$$
R\left(\bigcup_{k=0}^{m-1} P_{z, \xi, \eta, k}\right) \subset K \times K \times K \times K .
$$

Hence the inclusion (3.5) is equivalent to

$$
\begin{array}{r}
S_{z, \xi, \eta}=\bigcup_{k=0}^{m-1}\left\{-\cos \theta p_{1}+\sin \theta p_{3},-\cos \theta p_{2}+\sin \theta p_{4},-\sin \theta p_{1}-\cos \theta p_{3},\right. \\
\left.-\sin \theta p_{2}-\cos \theta p_{4}:\left(p_{1}, p_{2}, p_{3}, p_{4}\right) \in P_{z, \xi, \eta, k}\right\} \subset K .
\end{array}
$$

Now, we can choose $\alpha \in \mathbf{R}(\alpha \neq 0)$ such that

$$
\cos \frac{2 \pi k}{m}-\alpha \sin \frac{2 \pi k}{m} \neq 0, \sin \frac{2 \pi k}{m}+\alpha \cos \frac{2 \pi k}{m} \neq 0
$$

for all $k=0,1, \cdots, m-1$. Then it is easy to check that the set $S_{z, \xi, t+\alpha t i}$ is contained in the set of the form $U+t V$, where 


$$
\begin{aligned}
& U=\bigcup_{k=0}^{m-1}\left\{-\cos \theta\left(x+u \cos \frac{2 \pi k}{m}-v \sin \frac{2 \pi k}{m}\right),-\cos \theta\left(y+u \sin \frac{2 \pi k}{m}+\right.\right. \\
& \left.v \cos \frac{2 \pi k}{m}\right),-\sin \theta\left(x+u \cos \frac{2 \pi k}{m}-v \sin \frac{2 \pi k}{m}\right),-\sin \theta\left(y+u \sin \frac{2 \pi k}{m}\right. \\
& \left.\left.+\mathrm{v} \cos \frac{\pi k}{m}\right)\right\} \\
& \bigcup\{-x \cos \theta+u \sin \theta,-y \cos \theta+v \sin \theta,-(x \sin \theta+u \cos \theta),-(y \sin \theta \\
& +\mathrm{v} \cos \theta),-x \cos \theta,-y \cos \theta,-x \sin \theta,-y \sin \theta\} \\
& V=\bigcup_{k=0}^{m-1}\left\{\sin \theta, \alpha \sin \theta,-\cos \theta,-\alpha \cos \theta,-\cos \theta\left(\cos \frac{2 \pi k}{m}-\alpha \sin \frac{2 \pi k}{m}\right),\right. \\
& -\cos \theta\left(\sin \frac{2 \pi k}{m}+\alpha \cos \frac{2 \pi k}{m}\right),-\sin \theta\left(\cos \frac{2 \pi k}{m}-\alpha \sin \frac{2 \pi k}{m}\right),-\sin \theta\left(\sin \frac{2 \pi k}{m}\right. \\
& \left.\left.+\alpha \cos \frac{2 \pi k}{m}\right)\right\} .
\end{aligned}
$$

By (3.4), for given $z=x+i y, \xi=u+i v \in \mathbf{C}$ and $M>0$ there exists $t \geq M$ such that

$$
S_{z, \xi, t+\alpha t i} \subset U+t V \subset K
$$

Thus, $\Omega$ satisfies (3.3).

Theorem 3.3. There exists a set $\Omega \subset \mathbf{C}^{2}$ of Lebesgue measure zero such that if $f: \mathbf{C} \rightarrow F$ satisfies the inequality

$$
\left\|\sum_{k=0}^{m-1} f\left(z+b_{k} \xi\right)-m f(z)\right\| \leq \delta \text { for } \operatorname{all}(z, \xi) \in \Omega
$$

for all $(z, \xi) \in \Omega$, then there exists a unique mapping $q: \mathbf{C} \rightarrow F$ satisfying

$$
\sum_{k=0}^{m-1} q\left(z+b_{k} \xi\right)-m q(z)=0 \text { for all } z, \xi \in \mathbf{C} .
$$

such that

$$
\|f(z)-f(0)-q(z)\| \leq 3^{m} \delta \text { for all } z \in \mathbf{C}
$$


Proof. The proof follows immediately from Theorems 3.2 and 2.1.

Remark 3.4. It is easy to see that the set $\Omega_{d}=\left\{(z, \xi) \in \mathbf{C}^{2}:|z|+|\xi| \geq d\right\}$ also satisfies (3.3). Thus, the result of Theorem (3.3) holds when $\Omega$ is replaced by $\Omega_{d}$. Thus, as consequence of Theorem (3.3) with the above remark, we obtain a strong version of asymptotic behavior of $f$ satisfies

$$
\left\|\sum_{k=0}^{m-1} f\left(z+b_{k} \xi\right)-m f(z)\right\| \rightarrow 0, \text { as }|z|+|\xi| \rightarrow \infty, \quad(z, \xi) \in \Omega
$$

Corollary 3.5. Suppose that $f: \mathbf{C} \rightarrow F$ satisfies (3.6). Then $f$ satisfies the functional equation

$$
\sum_{k=0}^{m-1} f\left(z+b_{k} \xi\right)=m f(z), z, \xi \in \mathbf{C} .
$$

Secondly, we consider (3.2) in view of Corollary 2.2, it suffice to construct a set $\Omega \subset \mathbf{R}^{2}$ of measure zero satisfying (2.3).

Theorem 3.6. Let $\Omega=\exp (-i \theta) K \times K$ be the rotation of $K \times K$ by $-\theta$. Then $\Omega$ satisfies (2.3) and has two-dimensional Lebesgue measure 0.

Proof. The proof is similar to the proof of Theorem (3.2). However, we prove the completeness. Let $x, y, t \in \mathbf{R}$, and let

$$
P_{x, y, t}=\{(x+y, t),(x-y, t),(x+t, y),(x-t, y),(x, t)\} .
$$

Then $\Omega$ satisfies (2.3) if and only if, for every $x, y \in \mathbf{R}$ such that

$$
\exp (i \theta) P_{x, y, t} \subset K \times K \text {. }
$$

The inclusion (3.7) is equivalent to

$$
S_{x, y, t}:=\left\{-\cos \theta u+\sin \theta v,-\sin \theta u-\cos \theta v:(u, v) \in P_{x, y, t}\right\} \subset K .
$$

It is easy to check that the set $S_{x, y, t}$ is contained in a set of form $U+t V$, where

$$
\begin{aligned}
U= & \{\cos \theta(x+y), \sin \theta(x+y), \cos \theta(x-y), \sin \theta(x-y), x \cos \theta \\
& -y \sin \theta, y \cos \theta+x \sin \theta, x \cos \theta, x \sin \theta\} \\
V= & \{ \pm \sin \theta, ; \pm \cos \theta\}
\end{aligned}
$$


By Lemma 3.1, for given $x, y \in \mathbf{R}$ and $M>0$ there exists $t \geq M$ such that

$$
S_{x, y, t}=U+t V \subset K .
$$

Thus, $\Omega$ satisfies (2.3).

By Corollary 2.2 and Theorem (3.6) we have the following result.

Theorem 3.7. Let $\delta \geq 0$. There exists a set $\Omega \subset \mathbf{R}^{2}$ of Lebesgue measure zero such that if $f: \mathbf{R} \rightarrow F$ satisfies the inequality

$$
\|f(x+y)+f(x-y)-2 f(x)\| \leq \delta .
$$

only for all $x, y \in \Omega$, then there exists a unique additive mapping $A: \mathbf{R} \rightarrow F$ such that

$$
\|f(x)-f(0)-A(x)\| \leq 9 \delta \text { for all } x \in \mathbf{R} .
$$

: The authors are very thankful to the referee for valuable suggestions.

\section{References}

[1] T. Aoki, On the stability of the linear transformation in Banach spaces, Journal of the Mathematical Society of Japan, 2, pp. 64-66, (1950).

[2] J. A. Baker, A general functional equation and its stability, Proceeding of the American Mathematical Society, 133, pp. 1657-1664, (2005).

[3] N. Brillouet-Belluot, J. Brzdęk, K. Ciepliǹski, On some recent developments in Ulam's type stability, Abstract and Applied Analysis, (2012).

[4] J. Brzdęk, On a method of proving the Hyers-Ulam stability of functional equations on restricted domains, The Australian Journal of Mathematical Analysis and Applications, 6, pp. 1-10, (2009).

[5] A. B. Chahbi, A. Charifi, B. Bouikhalene and S. Kabbaj, Nonarchimedean stability of a Pexider K-quadratic functional equation, Arab Journal of Mathematical Sciences, 21, pp. 67-83, (2015). 
[6] A. Chahbi, M. Almahalebi, A. Charifi and S.Kabbaj Generalized Jensen functional equation on restricted domain, Annals of West University of Timisoara-Mathematics, 52, pp. 29-39, (2014).

[7] P. W. Cholewa, Remarks on the stability of functional equations, Aequationes mathematicae, 27, pp. 76-86, (1984).

[8] J. Chung, Stability of a conditional Cauchy equation on a set of measure zero, Aequationes mathematicae, 87, pp. 391-400, (2014).

[9] J. Chung and J. M. Rassias, Quadratic functional equations in a set of Lebesgue measure zero, Journal of Mathematical Analysis and Applications, 419, pp. 1065-1075, (2014).

[10] J. Chung and J. M. Rassias, On a measure zero Stability problem of a cyclic equation, Bulletin of the Australian Mathematical Society, 93, pp. 1-11, (2016).

[11] S. Czerwik, On the stability of the quadratic mapping in normed spaces, Abhandlungen aus dem Mathematischen Seminar der Universität Hamburg, 62, pp. 59-64, (1992).

[12] P. Găvruta, A generalization of the Hyers-Ulam-Rassias stability of approximately additive mappings, Journal of Mathematical Analysis and Applications, 184, pp. 431-436, (1994).

[13] D. H. Hyers, On the stability of the linear functional equation, Proceedings of the National Academy of Sciences, 27, pp. 222-224, (1941).

[14] D. H. Hyers and Th. M. Rassias, Approximate homomorphisms, Aequationes Mathematicae, 44, pp. 125-153, (1992).

[15] D. H. Hyers, Transformations with bounded $n$-th differences, Pacific Journal of Mathematics, 11, pp. 591-602, (1961).

[16] K. W. Jun and Y. H. Lee, A generalization of the Hyers-Ulam-Rassias stability of Jensen's equation, Journal of Mathematical Analysis and Applications, 238, pp. 305-315, (1999).

[17] S. M. Jung, On the HyersUlam stability of the functional equations that have the quadratic property, Journal of Mathematical Analysis and Applications, 222, pp. 126-137, (1998). 
[18] C. F. K. Jung, On generalized complete metric spaces, Bulletin of the American Mathematical Society, 75, pp. 113-116, (1969).

[19] R. Łukasik, Some generalization of Cauchy's and the quadratic functional equations, Aequationes Mathematicae, 83, pp. 75-86, (2012).

[20] A. Najati, S. M. Jung, Approximately quadratic mappings on restricted domains, Journal of Inequalities and Applications, (2010).

[21] J. C. Oxtoby, Measure and Category, Springer, New-York (1980).

[22] Th. M. Rassias, On the stability of linear mapping in Banach spaces, Proceedings of the American Mathematical Society, 72, pp. 297-300, (1978).

[23] Th. M. Rassias, On the stability of the functional equations and a problem of Ulam, Acta Applicandae Mathematicae, 62, pp. 23-130, (2000).

[24] Th. M. Rassias and P. Šemrl, On the behavior of mappings which do not satisfy Hyers-Ulam stability, Proceedings of the American Mathematical Society, 114, pp. 989-993, (1992).

[25] Th. M. Rassias, and J. Tabor, Stability of Mappings of Hyers-Ulam Type, Hardronic Press, (1994).

[26] J. M. Rassias, On the Ulam stability of mixed type mappings on restricted domains, Journal of Mathematical Analysis and Applications, 276, pp. 747-762, (2002).

[27] F. Skof, Local properties and approximations of operators, Rendiconti del Seminario Matematico e Fisico di Milano, 53, pp. 113-129, (1983).

[28] H. Stetkær, Functional equations involving means of functions on the complex plane, Aequationes Mathematicae, 56, pp. 47-62, (1998).

[29] S. M. Ulam, A Collection of Mathematical Problems, Interscience Publishers, 8(1960). 


\section{Hajira Dimou}

Department of Mathematics,

Faculty of Sciences, IBN Tofail University, BP: 14000, Kenitra,

Morocco

e-mail : dimouhajira@gmail.com

\section{Youssef Aribou}

Department of Mathematics, Faculty of Sciences, IBN Tofail University, BP: 14000, Kenitra,

Morocco

e-mail : aribouyoussef3@gmail.com

\section{Abdellatif Chahbi}

Department of Mathematics, Faculty of Sciences, IBN Tofail University, BP: 14000, Kenitra, Morocco

e-mail : ab-1980@live.fr

and

Samir Kabbaj

Department of Mathematics, Faculty of Sciences, IBN Tofail University, BP: 14000, Kenitra, Morocco

e-mail : samkabbaj@yahoo.fr 